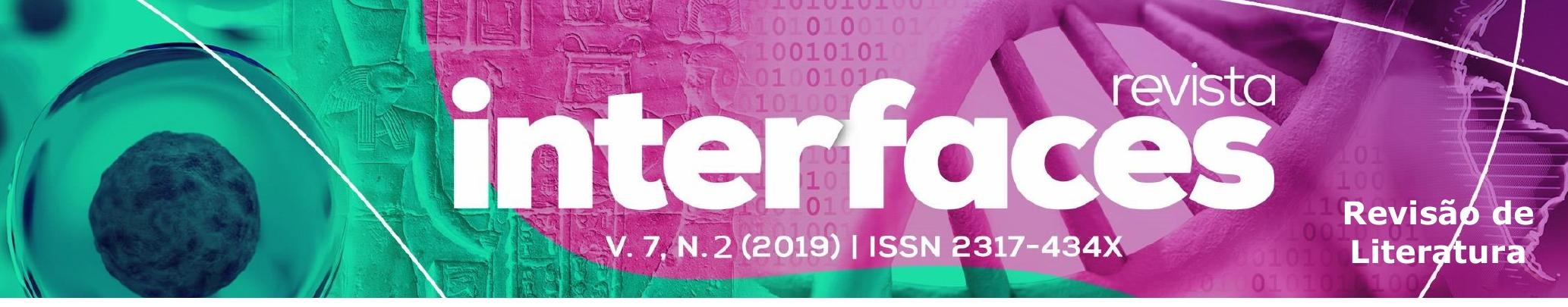

\title{
ABORDAGENS TERAPÊUTICAS NAS DOENÇAS INFLAMATÓRIAS: UMA REVISÃO
}

\author{
THERAPEUTIC APPROACHES IN INFLAMMATORY DISEASES: A REVIEW
}

\begin{abstract}
Priscilla Ramos Freitasa*, Johnatan Wellisson da Silva Mendes ${ }^{b}$, Kaio Jefté de Oliveira Diasc, Marcos Aurélio Nogueira de Carvalho Filhoc, Ana Carolina Justino de Araújoa , Jaime Ribeiro Filho ${ }^{\prime}$

Laboratório de Microbiologia e Biologia Molecular - URCA Laboratório de Pesquisa de Produtos Naturais - URCA Centro Universitário Leão Sampaio ${ }^{C}$ Instituto Gonçalo Moniz, Fundação Oswaldo Cruz (IGM-FIOCRUZ/BA) ${ }^{d}$ *E-mail: priscilla.r.freitas@hotmail.com
\end{abstract}

\begin{abstract}
RESUMO
O processo inflamatório é resultante de uma resposta do organismo a uma lesão tecidual com o objetivo de restaurar a homeostase. Inicialmente ocorre o recrutamento e a ativação de leucócitos, mas com a remoção do agente agressor, a resposta inflamatória é programada para cessar, o que chamamos de resolução da inflamação. O presente estudo consiste em uma revisão de literatura sobre as abordagens terapêuticas nas doenças inflamatórias. Em que teve como critérios de inclusão: estudos em inglês, português ou espanhol, cujo o tema central fossem relacionados ao processo inflamatório ou ao tratamento farmacológico destes processos, assim fazendo parte do presente estudo 47 produções selecionadas. Os anti-inflamatórios não-esteroidais (AINEs) e glicocorticoides (GC) são os fármacos mais utilizados para tratar as doenças inflamatórias. Em geral, estas drogas são eficazes para conter o processo inflamatório, porém possuem muitos efeitos colaterais que trazem riscos aos pacientes. Deste modo, a busca por novos compostos que promovam a resolução da inflamação e tragam menos efeitos colaterais aos pacientes é um desafio para as pesquisas voltadas para a farmacologia da inflamação
\end{abstract}

Palavras chave: Anti-inflamatórios; Glicocorticóides; Inflamação; Farmacologia.

\section{ABSTRACT}

The inflammatory process is the result of an organism's response to a tissue injury to restore homeostasis. Initially there is recruitment and activation of leukocytes, but with the removal of the offending agent, the inflammatory response is programmed to cease, which we call inflammation resolution. The present study consists of a literature review on therapeutic approaches in inflammatory diseases. In which had as inclusion criteria: studies in English, Portuguese or Spanish, whose central theme were related to the inflammatory process or pharmacological treatment of these processes, thus being part of the present study 47 selected productions. Non-steroidal anti-inflammatory drugs (NSAIDs) and glucocorticoids (GC) are the most commonly used drugs to treat inflammatory diseases. In general, these drugs are effective in containing the inflammatory process, but have many side effects that pose risks to patients. Thus, the search for new compounds that promote inflammation resolution and bring less side effects to patients is a challenge for research focused on the pharmacology of inflammation.

Keywords: Anti-inflammatory; Glucocorticoids; Inflammation; Pharmacology. 


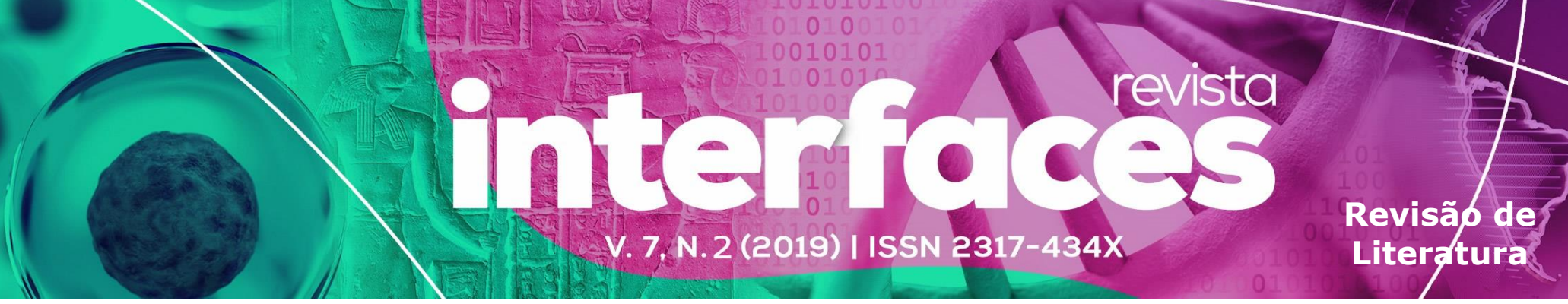

DALLE; RITTER, 2003).

Anticorpos monoclonais, inibidores de citocinas, antagonistas de receptores específicos e inibidores de quinases estão entre os fármacos mais recentemente desenvolvidos para o tratamento de doenças inflamatórias. Contudo, estes fármacos também possuem limitações de custo e limitações terapêuticas, como por exemplo, via de administração, danos gastrointestinais, hepáticos e renais (uso crônico) (MEDZHITOV, 2008). Este cenário revela a necessidade da busca por novos compostos para o tratamento de doenças inflamatórios.

As pesquisas na área da farmacologia têm aumentado consideravelmente nos últimos anos, assim, muitos pesquisadores estão em busca de novas substâncias bioativas extraídas de plantas medicinais, para desenvolver novos fármacos que possam ser utilizados no tratamento de doenças inflamatórias (MEGRAJ et al., 2011; SANTOS, 2006).

Um bom exemplo dessas pesquisas é a utilização de plantas para o tratamento de patologias, uma prática bastante antiga que, apesar do desenvolvimento das pesquisas modernas, ainda correspondem de 25 a $30 \%$ dos agentes terapêuticos de origem natural (MOTTA et al., 2013). Um exemplo deste potencial terapêutico é o Acheflan, um fármaco que possui ação anti-inflamatória em músculos e tendões. Este medicamento é encontrado na forma em gel ou em creme, o mesmo é obtido a partir do óleo essencial de Cordia verbenacea, popularmente conhecida como erva-baleeira (MICHIELIN, 2009).

\section{CONSIDERAÇÕES FINAIS}

De um modo geral, no cenário das doenças inflamatórias, as estratégias terapêuticas clássicas estão principalmente baseadas no uso de medicamentos anti-inflamatórios, cujo foco principal é diminuir ou neutralizar o nível de mediadores pró-inflamatórios e/ou impedir o recrutamento e ativação de leucócitos no sítio inflamatório Contudo, ultimamente tem sido reconhecido que estratégias baseadas na resolução da inflamação têm um grande potencial para o tratamento de inúmeras condições inflamatórias. A perspectiva é de que tais medicamentos possuam menos efeitos colaterais e, portanto, melhorem a qualidade de vida dos usuários.

\section{REFERÊNCIAS}

AKIRA, S. et al. Biology of multifunctional cytokines: IL 6 and related molecules (IL 1 and TNF). The FASEB journal, v. 4, n. 11, p. 2860-2867, 1990.
AMULIC, B. et al. Neutrophil function: from mechanisms to disease. ANNUAL REVIEW OF IMMUNOLOGY, v. 30, p. 459-489, 2012.

ANTI, S. M. A.; GIORGI, R. D. N.; CHAHADE, W. H. Antiinflamatórios hormonais: glicocorticóides. Einstein, v. 6, n. 1 , p. $159-165,2008$.

ANTONOW, D. R.; MONTEIRO, G. A.; ARAUJO, M. C. S. Glicocorticoides: uma meta-análise. Disciplinarum Scientia Saúde, v. 8, n. 1, p. 51-68, 2016.

AOKI, T.; NARUMIYA, S. Prostaglandins and chronic inflammation. Trends in pharmacological sciences, v. 33, n. 6, p. 304-311, 2012.

BAVARESCO, L.; BERNARDI, A.; BATTASTINI, A. M. O. Glicocorticóides: Usos clássicos e emprego no tratamento do câncer. Infarma, v. 17, n. 7, p. 9, 2005.

BRUNTON, L. L. Goodman \& Gilman: Las bases farmacológicas de la terapéutica 12 edição McGraw Hill Mexico, 2012.

CASTILHO, L. S.; RESENDE, V. L.S.; PAIXÃO, H. H. Os antiinflamatórios não-esteróides inibidores da síntese de prostaglandinas mais utilizados em Odontologia. Revista do Conselho Regional de Odontologia de Minas Gerais, v. 4, n. 1, p. 32-7, 1998.

COUTURE, R. et al. Kinin receptors in pain and inflammation. European journal of pharmacology, v. 429, n. 1-3, p. 161-176, 2001.

DE SOUZA, F. C. B.; DA SILVA, M. Z. M. Controle do processo inflamatório na odontologia com antiinflamatorios não-esteroidais. REVISTA UNINGÁ REVIEW, v. 20, n. 2, 2018.

ESTRADA, H. A. G.; RUIZ, K. N. G.; MEDINA, J. D. Actividad antiinflamatoria de productos naturales. Boletín Latinoamericano y del Caribe de Plantas Medicinales y Aromáticas, v. 10, n. 3, 2011.

FARSHCHI, A.; GHIASI, G. Comparison the analgesic effects of single dose administration of tramadol or piroxicam on postoperative pain after cesarean delivery. Acta Medica Iranica, v. 48, n. 3, p. 148-153, 2010. 
